\title{
SIMULATION OF LINE SCALE CONTAMINATION IN CALIBRATION UNCERTAINTY MODEL
}

\author{
Druzovec, M. ; Acko, B. ${ }^{* *}$; Godina, A. ${ }^{* *} \&$ Welzer, T. \\ ${ }^{*}$ University of Maribor, Faculty of Electrical Engineering and Computer Science \\ ${ }^{* *}$ University of Maribor, Faculty of Mechanical Engineering \\ ${ }^{* / * *}$ Smetanova 17, SI-2000 Maribor, Slovenia \\ E-Mail: marjan.druzovec@uni-mb.si
}

\begin{abstract}
Precise calibration of quartz line scales is very important for assuring traceability of microscopic measurements. Very significant influence in calibration uncertainty budget is represented by uncertainty of line centre detection. Line centre is usually detected through video signal processing using different types of algorithms. This paper is presenting calibration procedure that was developed in the Laboratory for Production Measurement at the Faculty of Mechanical Engineering in Maribor. It is focused in uncertainty analysis and especially in the influence of line scale contamination on determination of line centre position. Different types of line scale contamination like dirt spots, scratches, line edge incorrectness, and line intensity were simulated in order to test the ability of the line centre detection algorithm to eliminate such influences from the measurement results.

(Received in June 2007, accepted in May 2008. This paper was with the authors 5 months for 2 revisions.)
\end{abstract}

Key Words: Line Scale, Calibration, Uncertainty, Video-Positioning System, Simulation

\section{INTRODUCTION}

Laboratory of Production Measurement at the University of Maribor is designated national metrology laboratory for length within the National metrology institute (MIRS). In this role we are researching industrial and scientific needs regarding the calibration of length quantities in Slovenia. Increasing needs in the field of line scale calibrations initiated the development of a new measurement set-up some years ago. Our goal was to reach best calibration and measurement capability (CMC) within 100 nanometers. Due to limited financial resources we decided to reconstruct our existing Zeiss universal length machine and to use it together with the Agilent laser interferometer and a new video positioning system consisting of a CCD camera and an optical zoom microscope.

The first stage of our research was focused in the line centre detection. One of the very important influences on measurement uncertainty is contamination of the scale lines. Every spot, dirt part etc. can cause an error in line centre calculation that is performed with our algorithm.

This paper introduces a simulation approach to determining contamination influences on the measuring uncertainty. The simulations were applied in order to show robustness of our algorithm, which shall be demonstrated by insignificant influences of line contamination on total measuring uncertainty.

\section{MEASURING SYSTEM}

\subsection{Description of the measuring system}

The measuring system for calibrating line scales is shown in Fig. 1, and its schematic diagram in Fig. 2. It is actually a set-up that integrates a moving table, laser interferometer (LI), video- 
positioning system (VPS), and a line scale. It is designed in such a way that the Abbe error is minimised [1, 2]. The line scale is a two-dimensional artefact (Fig. 3) used for length measurements along one axis, called the line scale axis. Measurement distances on the line scale are marked with measuring lines (scale marks), which are positioned perpendicularly to the line scale axis. The scale is fixed on the moving table that moves to a new position for every measurement. The movement direction is actually physical measuring direction and should be parallel to the line scale axis. The laser interferometer measures the position of the line scale, while the video-positioning system determines the position of the observed line on the images taken by the CCD camera [3, 4].

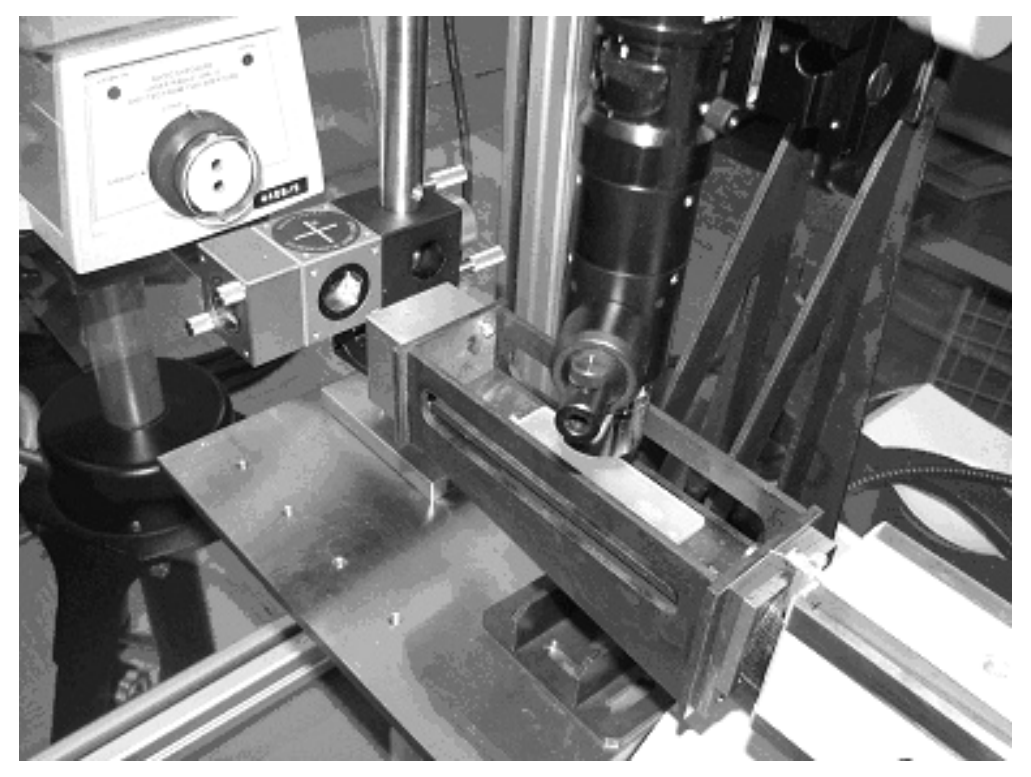

Figure 1: Measuring system set-up.

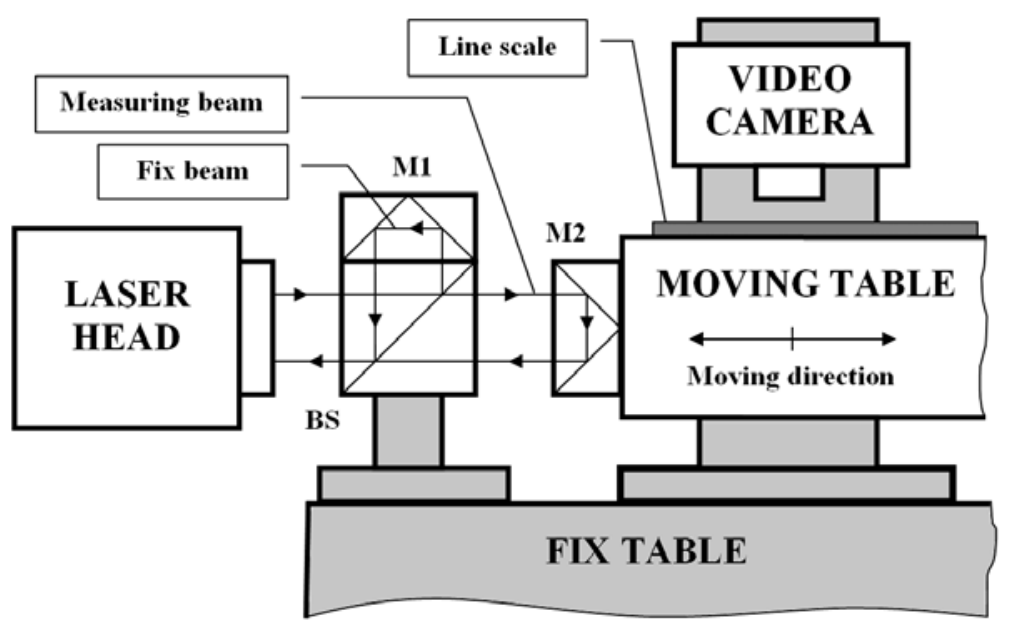

Figure 2: Schematic diagram of the measuring system.

A reference line (usually "zero line") is chosen to define the zero position of the line scale for LI and the reference position on the images taken by a CCD camera of the VPS. The measurement task is to measure the distance between the reference line and any line of interest, called a measured line, which appears in the image after moving the line scale to the measurement position. The laser interferometer measures the distance between the reference line and the reference position on the image. VPS doesn't only serve to indicate the measured 
line, but also to determine its distance from the reference position to actual position of the measured line in the image. Final measurement result is a sum of measured line-scale movement (LI indication), and the offset of the line in the image (VPS indication).

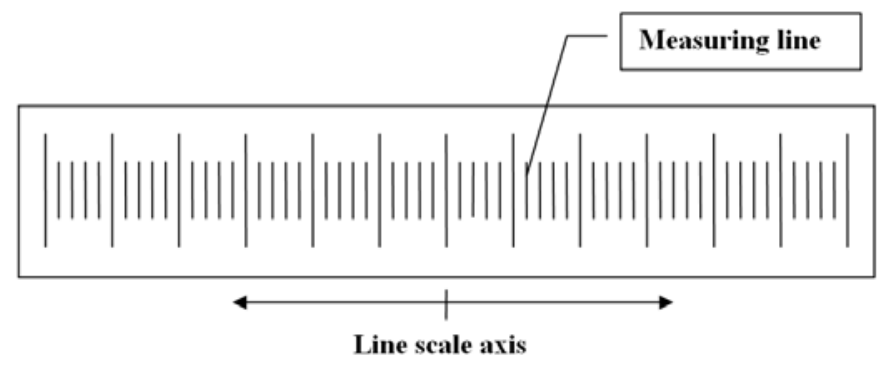

Figure 3: Line scale.

\subsection{Laser interferometer}

Laser interferometer measurement system is a standard instrument for precise length measurements [3-6]. It consists of electronic devices and optical elements. We use HP 5528 laser interferometer with resolution set to $10 \mathrm{~nm}$ and with standard optical components [6]. Special mounting elements for the optics were developed for the instrument used for calibration. A schematic diagram of the LI and the positions of the optical components can be seen in Fig. 2. Optical elements are set in such way, that the LI measures instantaneous position of the moving table.

\subsection{Video-positioning system}

The video-positioning system consists of a zoom microscope and a CCD video camera connected to a computer via fireware. The CCD camera takes images of the line scale and sends them to the computer. Software analyzes the images and determines the position of the measured line in the image, as well as its distance from the reference position in pixels.

The image on the VPS screen (Fig. 4) merges the image of a line scale taken by the CCD camera, and the computer-animated image that shows measurement window, reference position and line position.

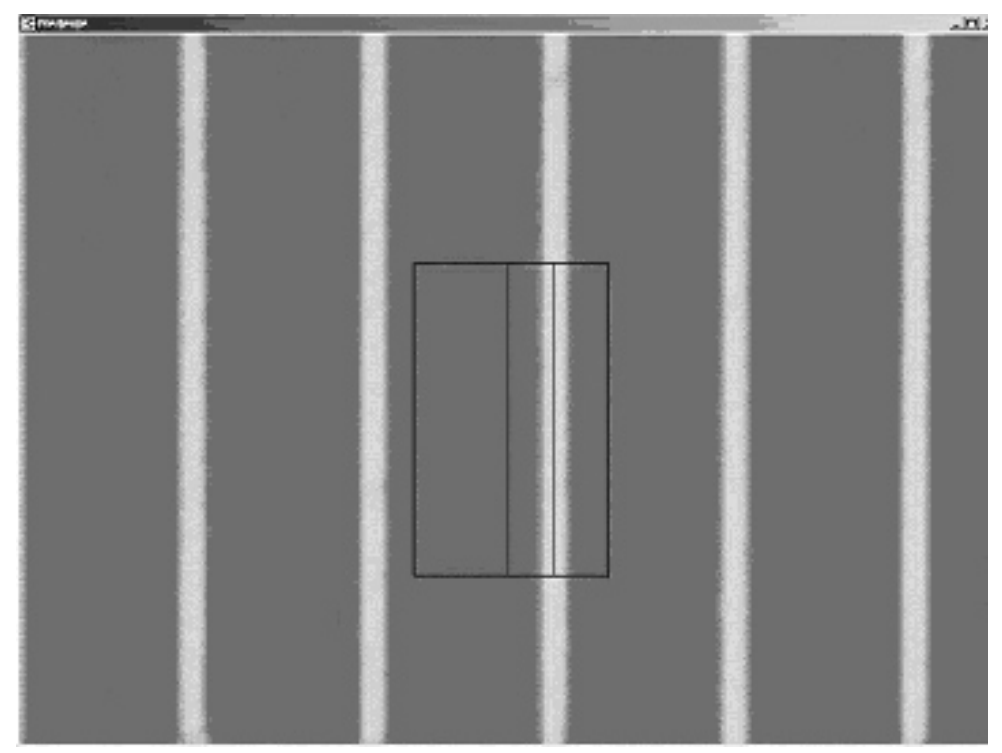

Figure 4: VPS screen image. 
- Video image is a video camera snapshot of a line scale. The CCD camera takes 25 monochrome images per second in resolution 800x600 pixels. VPS analyzes all images in real-time.

- Measurement window is represented as a rectangular frame on the VPS screen. The size of the frame and its position is defined by a mouse interactively on the VPS screen by the operator at the beginning of the measurement The image processing system only analyzes the content of the measurement window, so the size of the frame must be limited in content to only one measured line.

- Reference position is marked with a fix vertical line in the measurement window. Its position is defined during the initialization of the measuring system. The best position is approximately in the centre of the measurement window, but it is unnecessary to position it very precisely. The laser interferometer measures the length between the reference line on the line scale and the reference position on the image.

- Line position is marked with a vertical line in the centre of the single measured line inside the measurement window. It represents a measured line limited to one pixel in width, and marks its current position. The video-positioning system actually determines the distance between the line position and the reference position in pixels.

\section{MEASURING PROCEDURE}

The measuring system must be initialized before the measurement. Initialization includes adjustment of the measuring system's set-up, resolution determination, and setting a measurement window.

\subsection{Adjustment of the measuring system}

The measuring system is a set-up that incorporates a moving table, laser interferometer, video-positioning system, and a line scale. In order to make the measurement possible, all these components must be adjusted according to the measuring direction, which is defined by the movement direction of the table.

\subsection{Resolution determination}

Resolution or scale factor is a numerical value of pixel size in millimeters. It is determined by moving a selected line across the screen. LI measures the actual line movement $L_{r e s}$ in mm, while the VPS estimates the same distance $P_{\text {res }}$ in the image in pixels. The resolution $R$ is then denoted by:

$$
R=L_{r e s} / P_{r e s}[\mathrm{~mm} / \mathrm{pixel}]
$$

\subsection{Setting the measurement window and zero position}

In the last step before measuring, the operator draws a measurement window approximately in the middle of the image. Using the vertical position and the height of the measurement window, the operator chooses the segments of the scale lines in which the measurement will be performed. Then the reference line has to be moved approximately to the centre of the measurement window. This position serves for initializing the VPS and LI. The current position of the reference line simultaneously defines the reference position $s_{r e f}$ on the image of the VPS, and the origin value $L_{\text {LIzero }}$ for the zero position in the LI counting system. 


\subsection{Measurement}

The measurement goal is to measure distances between the reference line and chosen measuring lines on the line-scale. For every measurement result, the moving table is moved into a new position, in such way that the measured line is inside the measurement window. The laser interferometer measures the distance $L_{L I}$ between the reference line and the reference position on the image. And, the video-positioning system estimates the number of pixels between the measured line and the reference position. The number of pixels $P$ is converted into distance $L_{v}$ by:

$$
L_{v}=R \cdot P
$$

The final measurement result $L$ for every chosen measuring line is the sum of both measured values:

$$
L=L_{L I}+L_{v}-L_{\text {LIzero }}
$$

\subsection{Influences on line centre determination}

The intensity set is a deciding factor for estimating the position of the measurement-line. Its shape reflects the characteristics of an image in a measurement window and depends, not only upon the line of interest, but also some possible incorrectness of a line scale's surface (dirt, scratches, wear). For estimating the quality of the positioning procedure, we have to analyze how the image quality and the stripe quality influence positioning. The position of the stripe is computed from the intensity-set, so we have to analyze how the irregularities influence it.

Generally speaking, the intensity set shows average image brightness in the measurement window in corresponding $\mathrm{x}$-positions. Along the $\mathrm{x}$-axis the image is quite regular; it shows either a monotonous background or a monotonous stripe. So the intensity set (graph in Fig. 5) has only two different, almost constant, levels. The lower level represents the background and the peak of higher level represents the stripe. Difference between the levels depends on image contrast. The quality of the scale-mark also influences the intensity set. For instance, the wear of the line scale or dirt on it, reduces the image contrast at the edges of the stripe and, consecutively, the steepness of the peak in the intensity set.

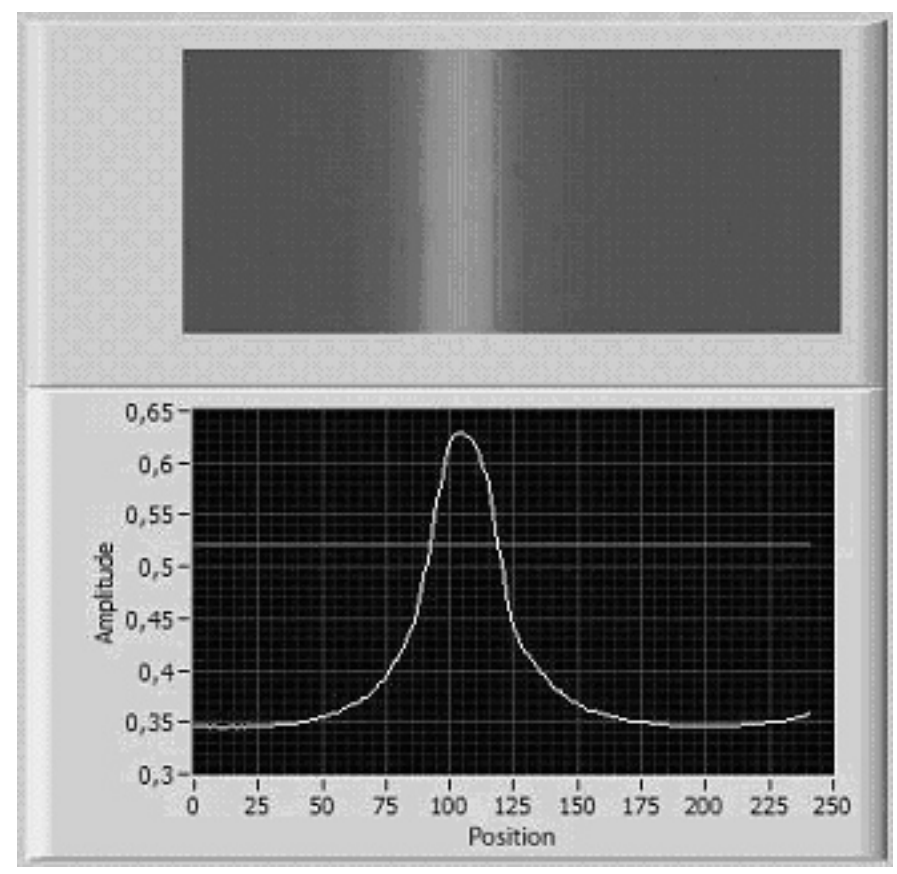

Figure 5: Image of a measurement window, and a graph of its intensity set. 
A serious change in the intensity set is the change that influences the positioning of the measured line. It should be an additional peak that crosses the level-line or non-monotonous change in steepness at the point where the intensity set crosses the level-line. Scratches and dirt cover a very small surface on a line scale and they can't cause any significant changes. The low and high levels of the intensity set remain almost constant, even in the case of incorrectness of the surface. This incorrectness could influence positioning only for very small image contrast, when the level of the peak in the intensity set is smaller. But, the system also controls a number of peaks that cross the level line and warns about error, if it senses more than one peak. In a normal case, the intensity set can only have one peak caused by a stripe that crosses the level line.

Possible disadvantage factors could be irregularities on a line scale (stain, dirt, scratches, rust, ...) and a surface that causes low-contrast between the stripe and the background on the image. Smaller contrast in scale-marks or the smaller sharpness of an image reduces the contrast at the edges of the stripe, and consecutively, the steepness of the peak in the intensity set. But this does not influence the positioning of the measured line, because this changes both edges in the same way, and the position line remains at the same position at the centre of the peak.

\section{UNCERTAINTY OF MEASUREMENT}

\subsection{Mathematical model of measurement}

The measured value is calculated as a sum of the laser interferometer and the videopositioning system indications (3). Deviation $e$ of the measured value $L$, therefore is given by:

$$
e=L_{L I}\left(1-\alpha_{m} \cdot \theta_{m}\right)-L_{n}+\delta L_{p 0}-\delta L_{p m p}+\delta L_{c o s}-\delta L_{d p}-\delta L_{p}-\delta L_{\text {Abbe }}-\delta L_{h}-\delta L_{r}
$$

where:

$e \quad$ - deviation of the line distance (calibration result) at $20{ }^{\circ} \mathrm{C}$

$L_{L I} \quad$ - corrected length measured by LI

$\alpha_{m} \quad$ - linear temperature expansion coefficient of the line scale

$\theta_{m} \quad$ - temperature deviation of the line scale from $20^{\circ} \mathrm{C}$

$L_{n} \quad$ - nominal length (without uncertainty)

$\delta L_{p 0} \quad$ - positioning error at zero position (influence of video position algorithm)

$\delta L_{p m p} \quad$ - positioning error at measuring position (influence of video position algorithm)

$\delta L_{\text {cos }} \quad$ - cosine error and errors of line scale alignment

$\delta L_{d p} \quad$ - dead path error

$\delta L_{p} \quad$ - scale compression due to the air pressure

$\delta L_{A b b e} \quad$ - error due to Abbe offsets; pitch and yaw

$\delta L_{h} \quad$ - effect of flatness deviation of the scale and microscope alignment

$\delta L_{r} \quad$ - effect of random influences

\subsection{Standard uncertainties}

For uncorrelated input quantities, the square of the standard uncertainty associated with the output estimate $y$ is given by equation (5) $[7,8,12]$.

$$
u^{2}(y)=\sum_{i=1}^{N} u_{i}^{2}(y)
$$


The quantity $u_{i}(y)(i=1,2, \ldots, N)$ is the contribution to the standard uncertainty associated with the output estimate $y$ resulting from the standard uncertainty associated with input estimate $x_{i}[12]$.

$$
u_{i}(y)=c_{i} \cdot u\left(x_{i}\right)
$$

Coefficient $c_{i}$ is the sensitivity coefficient associated with the input estimate $x_{i}$, i.e. the partial derivative of the model function $f$ with respect to $X_{i}$, evaluated at the input estimates $x_{i}$ [9-12].

$$
c_{i}=\frac{\partial f}{\partial x_{i}}=\left.\frac{\partial f}{\partial X_{i}}\right|_{X_{1}=x_{1} \ldots X_{N}=x_{n}}
$$

\begin{tabular}{|c|c|c|c|c|c|}
\hline $\begin{array}{c}\text { Value } \\
X_{i}\end{array}$ & $\begin{array}{l}\text { Estimated } \\
\text { value }\end{array}$ & $\begin{array}{c}\text { Standard } \\
\text { uncertainty }\end{array}$ & Distribution & $\begin{array}{l}\text { Sensitivity } \\
\text { coefficient }\end{array}$ & $\begin{array}{l}\text { Uncertainty } \\
\text { contribution }\end{array}$ \\
\hline$L_{L I}-C^{*}$ & $0 \mathrm{~mm}$ & $10 \mathrm{~nm}$ & normal & 1 & $10 \mathrm{~nm}$ \\
\hline$L_{L I}-L^{* *}$ & $\mathrm{~L}$ & $2.5 \cdot 10^{-7} \cdot L$ & normal & 1 & $2.5 \cdot 10^{-7} \cdot L$ \\
\hline$\alpha_{m}$ & $5 \cdot 10^{-7} \mathrm{~K}^{-1}$ & $10^{-7} \mathrm{~K}^{-1}$ & rectangular & $-\mathrm{L} \cdot 1 \mathrm{~K}$ & $-10^{-7} \cdot L$ \\
\hline$\theta_{m}$ & $0 \mathrm{~K}$ & $0.03 \mathrm{~K}$ & normal & $-\mathrm{L} \cdot 5 \cdot 10^{-7} \mathrm{~K}^{-1}$ & $-1.5 \cdot 10^{-8} \cdot L$ \\
\hline$\delta L_{p 0}$ & $0 \mathrm{~nm}$ & $15 \mathrm{~nm}$ & rectangular & 1 & $15 \mathrm{~nm}$ \\
\hline$\delta L_{p m}$ & $0 \mathrm{~nm}$ & $15 \mathrm{~nm}$ & rectangular & -1 & $-15 \mathrm{~nm}$ \\
\hline$\delta L_{\cos }$ & $0 \mathrm{~nm}$ & $2.5 \cdot 10^{-7} \cdot L$ & rectangular & 1 & $2.5 \cdot 10^{-7} \cdot L$ \\
\hline$\delta L_{d p}$ & $0 \mathrm{~nm}$ & $1.2 \mathrm{~nm}$ & rectangular & -1 & $-1.2 \mathrm{~nm}$ \\
\hline$\delta L_{p}$ & $0 \mathrm{~nm}$ & $6 \cdot 10^{-9} \cdot L$ & rectangular & -1 & $6 \cdot 10^{-9} \cdot L$ \\
\hline$\delta L_{A b b e}$ & $0 \mathrm{~nm}$ & $20 \mathrm{~nm}$ & rectangular & -1 & $-20 \mathrm{~nm}$ \\
\hline$\delta L_{h}$ & $0 \mathrm{~nm}$ & $8 \mathrm{~nm}$ & normal & -1 & $-8 \mathrm{~nm}$ \\
\hline$\delta L_{r}$ & $0 \mathrm{~nm}$ & $30 \mathrm{~nm}$ & normal & -1 & $-30 \mathrm{~nm}$ \\
\hline & & & & Total: & $\sqrt{(44 \mathrm{~nm})^{2}+\left(3.7 \cdot 10^{-7} \cdot L\right)^{2}}$ \\
\hline
\end{tabular}

Table I: Uncertainty budget for calibration of quartz line scales.

* - constant part

** - length dependent part

\subsection{Combined standard uncertainty and expanded standard uncertainty}

Combined standard uncertainty, calculated from the uncertainty budget, is:

$$
u=\sqrt{(44 \mathrm{~nm})^{2}+\left(3.7 \cdot 10^{-7} \cdot L\right)^{2}}
$$

The expanded uncertainty for the coverage factor $k=2$ is then:

$$
u=\sqrt{(88 \mathrm{~nm})^{2}+\left(7.4 \cdot 10^{-7} \cdot L\right)^{2}}
$$

\section{SIMULATION OF LINE CONTAMINATION}

Line scale contamination was simulated on a 500 pixels wide model line. This line was simulating typical object micrometer line at the highest possible magnification, for which best measurement capability can be reached. At the beginning of the simulation we have chosen fixed height and with of the evaluation window. Contamination samples were divided into white spots (a part of the line is missing) and dark spots (dust, scratches, etc.) with different height and width. The height was evaluated in \% of the chosen window height. The average 
line centre was determined for an ideal line in the beginning of each simulation step. After that the line was spoiled with a spot. The shift of the average line centre was calculated as indicated in Fig. 6.

Line edges detection

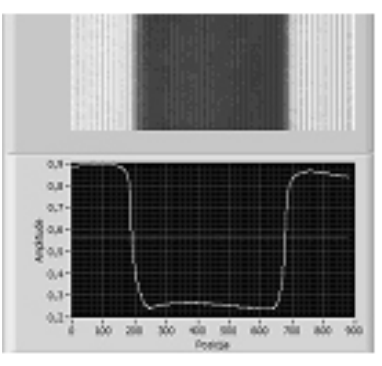

Line centre determination

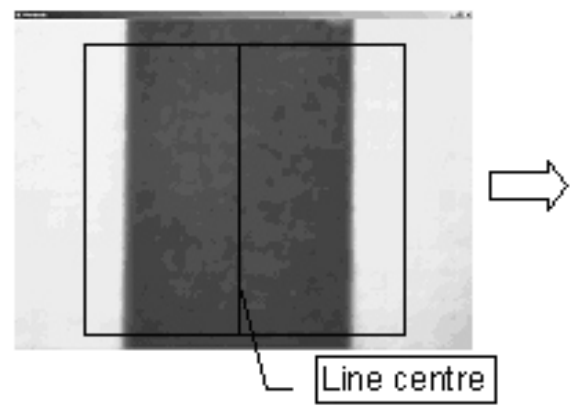

Line centre shift due to a spot

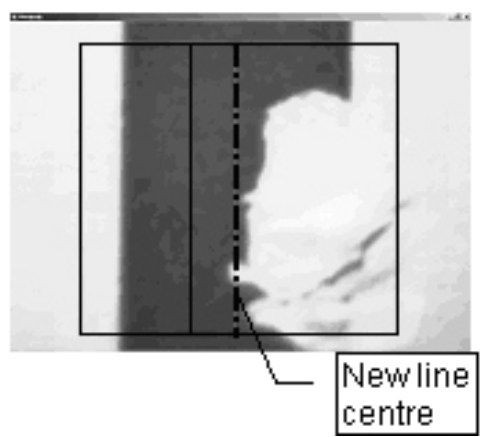

Figure 6: Contamination simulation procedure.

\subsection{Bright spot simulation}

About hundred bright spots were simulated on one edge, on both edges and in the middle of the line. As it was expected, one-sided spots caused the most significant line shift. Therefore, only such spots are presented in Table II. Two parameters are given to each spot: portion of the evaluation window height in \% and the average centre line shift in pixels.

Table II: Sample bright spot simulations.
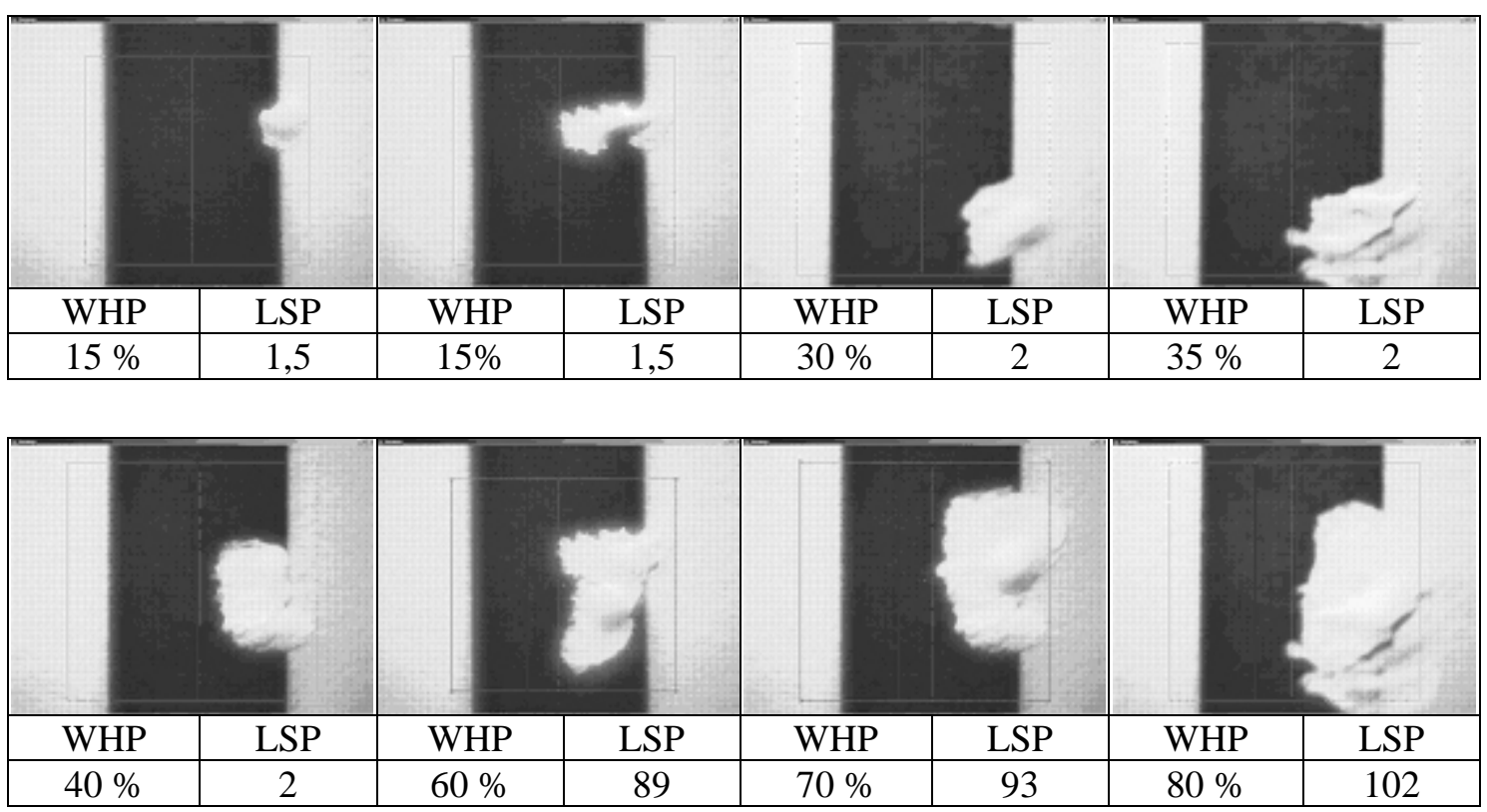

WHP: evaluation window height portion of the simulated spot

LSP: lines shift in pixels

\subsection{Dark spot simulation}

Dark spot simulation was performed in exactly the same way as the bright spot simulation. Sample results are shown in Table III. 
Table III: Sample dark spot simulations.
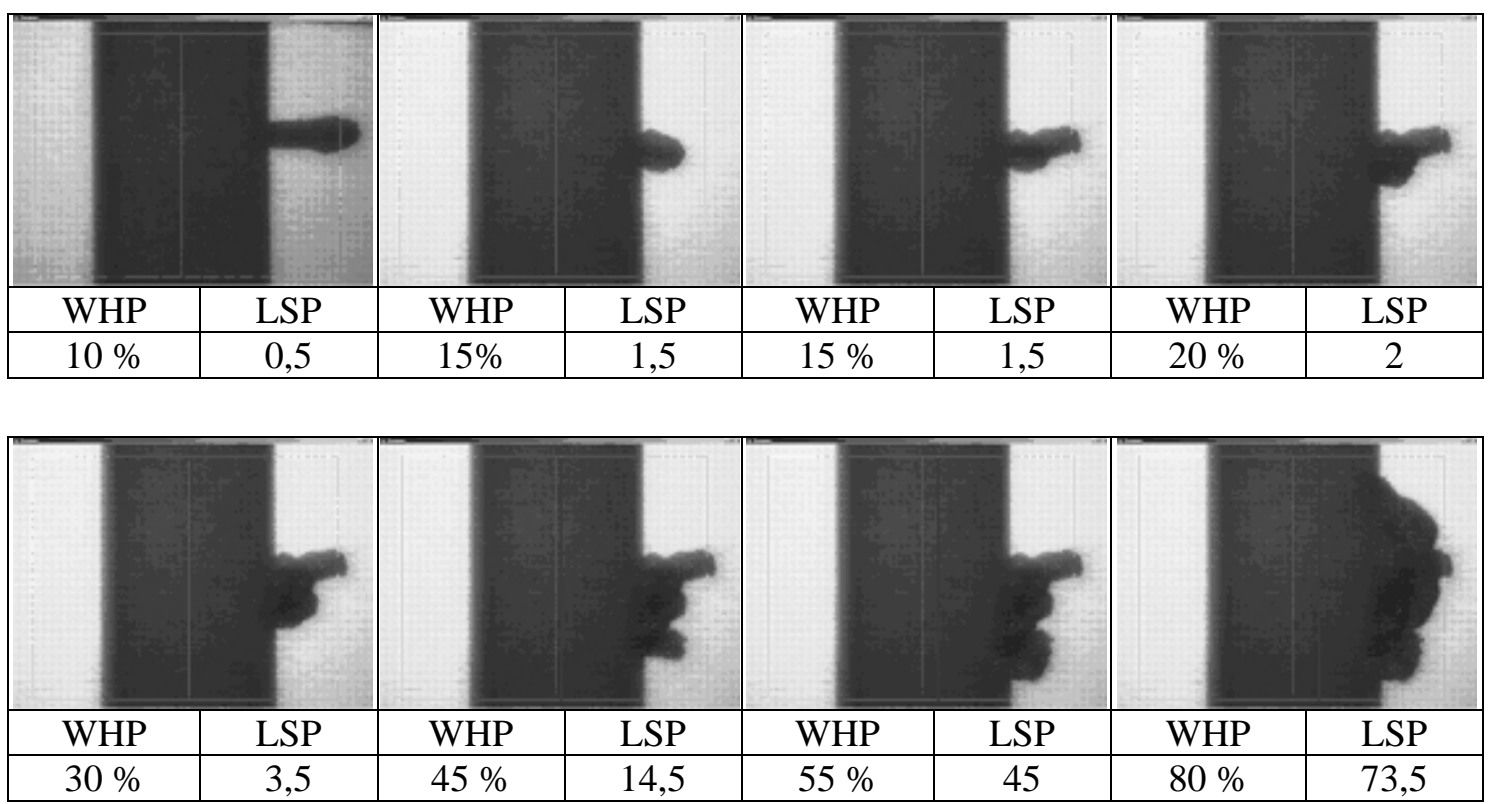

WHP: evaluation window height portion of the simulated spot

LSP: lines shift in pixels

\subsection{Analysis of the results}

Contamination of the line can be shown either as a black or a bright spot of the line or a combination of both. We have studied only a black line on a bright background. It can be assumed that a bright line on a dark background would behave in the same way. Already from the line centre calculation algorithm it is obvious that one-sided contamination will have more effect on the line centre shift than symmetrical or central contamination. This assumption was confirmed by the simulation. The main purpose of this simulation was to determine the relation between the height and the width of a contamination spot and the line centre shift. The results for a bright contamination spot are shown in Fig. 7, while Fig. 8 is showing the results for a dark spot. The left diagrams are presenting the influence of the spot height and the right diagrams the influence of the spot width at selected height. The results in the left diagrams are averages at each line height calculated from 15 different spot widths.
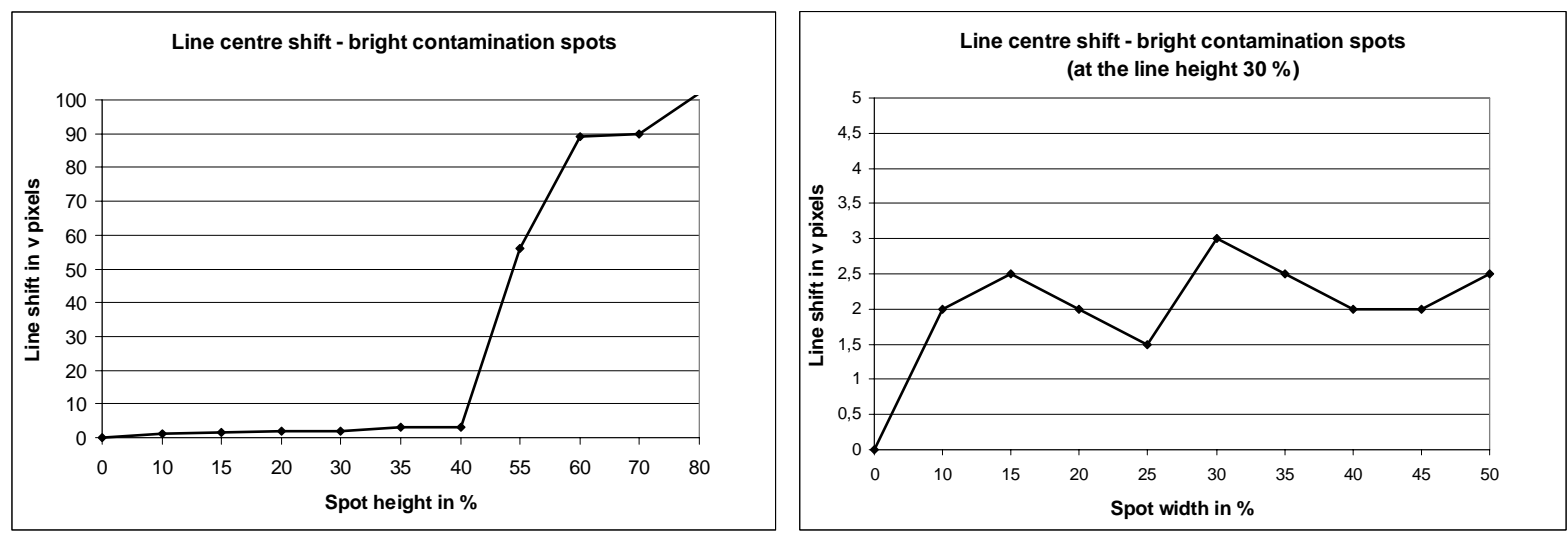

Figure 7: Line centre shift due to a bright contamination spot. 
The results in the right diagram show that the influence of the spot width is negligible. Line shift scatters randomly. However, the left diagram indicates significant influence of the spot height, especially when it exceeds $40 \%$ of the line width. Taking into account that the pixel dimension in the best measurement conditions $[9,11]$ corresponds to $53 \mathrm{~nm}$, the line shift of 3 pixels (at $40 \%$ spot height) is equal to $159 \mathrm{~nm}$. This value is not negligible in the total uncertainty budget $[7,12]$.
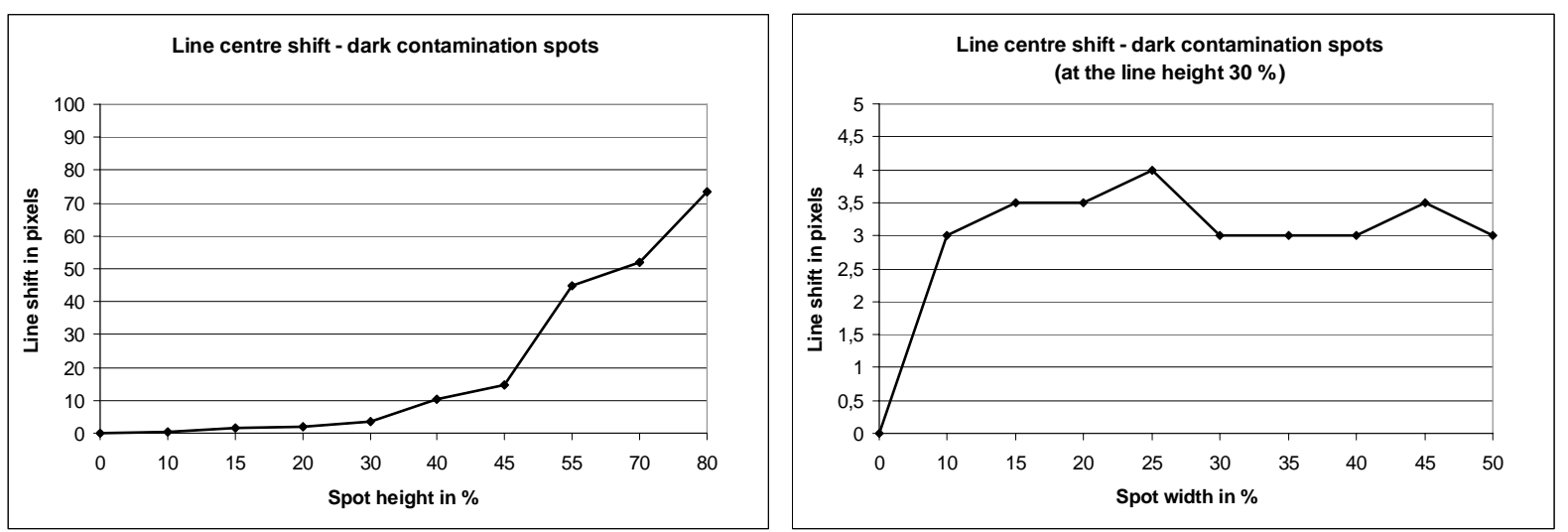

Figure 8: Line centre shift due to a dark contamination spot.

The results for the dark contamination spot are very similar to those for the bright spot. However, the line shift at $40 \%$ spot height is already significantly higher than the result for a bright spot.

From the above results it can be concluded, that the best measurement capability remains within the defined limits if the height of one-sided contamination spot is not exceeding $15 \%$ of the selected evaluation window height. Therefore, it is recommended to make the evaluation window as high as possible.

\section{CONCLUSION}

The intensity set (Fig. 5) is the deciding factor for estimating the centre of a measuring line. It shows the average image brightness in a measurement-window at a corresponding position in the measuring-direction. Any contamination (scratches, dirt, rust, stain, etc.) usually covers a small surface and it can't cause any serious change in the intensity set. Any dirt or damages on the line scale could reduce the sharpness of the measuring line at the edges on the image. Consecutively the steepness of the peak in the intensity set is reduced. However, this usually happens at both edges of the stripe and doesn't change the calculated position. Critical cases of one-sided contamination can be detected through the shape of the intensity set. It would get an asymmetrical form.

The performed simulation confirms the predicted robustness of the line centre determination algorithm for "normal" contamination levels. However, it was shown that any contamination spot exceeding $15 \%$ of the evaluation window height significantly influences the uncertainty budget. On the other hand, such contamination can be easily visually detected during the measurement and the line shift can be compensated. The results of this simulation will primarily help us to determine, which contamination level is significantly influencing the total uncertainty of measurement. In such cases we will be able to decide how the uncertainty shall be increased in respect to the line quality.

Further development of the line centre algorithm will be focused into sub-pixel line centre determination by advanced intensity set evaluation. Such algorithm could also comprise a 
module for calculating centre line shift due to contamination. This calculation will be based on experimental standard deviation of the normal symmetrical shape of the intensity set.

\section{ACKNOWLEDGMENTS}

This project was co-financed by the Ministry of Higher Education, Science and Technology Metrology Institute of Republic of Slovenia (MIRS) in the frame of the contract 6401$17 / 2007 / 12$ on performing activities and fulfilling obligations of the holder of the national standard of length.

\section{REFERENCES}

[1] Howick, E. F.; Sutton, C. M. (2001). Development of an automatic line scale measuring instrument, Proceedings of the SPIE - the International Society for Optical Engineering, 112-119

[2] TaeBong. E.; Jin, W. H. (2001). A precision length measuring system for a variety of linear artefacts, Measurement Science \& Technology, Vol. 12, No. 6, 698-701, doi: 10.1088/0957$\underline{0233 / 12 / 6 / 307}$

[3] Fujima, I.; Fujimoto, Y.; Sasaki, K.; Yoshimori, H.; Iwasaki, S.; Telada, S.; Matsumoto, H. (2003). Laser interferometer for calibration of a line scale module with analog output, Proceedings of SPIE - the International Society for Optical Engineering, 103-110

[4] Gonzalez, H.; Galvan, C.; Munoz, J. A. (2003). Processing Automatic Interferometric Calibration System for Line Scales, Proceedings of SPIE - the International Society for Optical Engineering, 93-102

[5] Luo, P. F.; Pan, S. P.; Chu, T. C. (2004). Application of computer vision and laser interferometer to the inspection of line scale, Optics and Lasers in Engineering, Vol. 42, No. 5, 563-584

[6] Acko, B.; Sostar, A. (1998). Uncertainty of measurement in calibration of one-coordinate measuring device with laser interferometer, Proceedings of the 9th International DAAAM symposium, 001-002

[7] Acko, B. (2003). A universal model for evaluating measuring uncertainty in calibration, International Journal of Simulation Modelling, Vol. 2, No. 4, 121-129

[8] Grabe, M. (2001). Estimation of measurement uncertainties - an alternative to the ISO Guide, Metrologia, Vol. 38, No. 2, 97-106, doi: 10.1088/0026-1394/38/2/1

[9] Taylor, B. N.; Kuyatt, C. E. (1994). Guidelines for Evaluating and Expressing the Uncertainty of NIST Measurement Results, NIST Technical Note 1297, U.S. Government Printing Office, Washington

[10] Willink, R.; Hall, B. D. A. (2002). Classical method for uncertainty analysis with multidimensional data, Metrologia, Vol. 39, No. 4, 361-370, doi: 10.1088/0026-1394/39/4/5

[11] Kacker, R.; Jones, A. (2003). On use of Bayesian statistics to make the Guide to the Expression of Uncertainty in Measurement consistent, Metrologia, Vol. 40, No. 5, 235-248, doi: 10.1088/0026-1394/40/5/305

[12] EA Publication (1999). Expression of the Uncertainty of Measurement in Calibration, EA document EA-4/02 